\title{
BACHELARD, ETAT DES LIEUX 55 ANS APRES SA MORT
}

\author{
BACHELARD, SITUAÇÃO DOS TRABALHOS 55 ANOS DEPOIS DE SUA \\ MORTE
}

Jean-Jacques Wunenburger ${ }^{1}$

\section{RESUMÉ}

L'article traite de la répercussion de la philosophie de Gaston Bachelard 55 ans après sa mort. Par son rationalisme, la pensée de Bachelard a une influence importante sur l'epistémologie française contemporaine. En guise de rêverie poétique, le bachelardisme refait surface au XXI e siècle à travers des interprétations artistiques, géographiques et architecturales. Le résultat de cette exposition vise alors place le lecteur dans ce que l'auteur appelle une double culture, dans l'opposition et la complémentarité des deux faces de l'esprit, animus et anima, donnat la profondeur humnaine d'être et les moyens de la liberté, la vérité e bonner. En même temps, se déploie ce bachelardisme trois neouvelles perspectives : léducation, l'écologie et la critique littéraire, que de nouveaus visages de la pensée d'aujourd'hui de ce grand penseur transdisciplinaire que traverse le corps à l'esprit, les affections de la sphère sociale. La critique littéraire nous conduit á la nouvelle herméneutique et à la phénoménologie que circulent autour de cette pédagogie esthétique de l'inteligence. Enfin, une écologie du paysage, òu l'imagination est une géo-pathos, à savoir un amour de la terre et être compris comme un écosystème intégré et actif dans un biomimétisme symbiotique entre l'homme et la nature.

Mots-clés: Gaston Bachelard. Héritage contemporain. Théorie-pratique. Double culture.

\footnotetext{
${ }^{1}$ Presidente da Associação Internacional Gaston Bachelard, da Associação dos Amigos de Gilbert Durand e do Centro Internacional de Pesquisas sobre o Imaginário (CRI2i). Professor de Filosofia Geral e das Imagens - Université JeanMulin - Lyon 3 (France). Vice-Líder do Imaginalis - Grupo de Estudos sobre Comunicação e imaginário PósGraduação em Comunicação e Informação e da Graduação em Comunicação Social da UFRGS
} 


\section{RESUMO}

O artigo trata da repercussão da filosofia de Gaston Bachelard 55 anos após a sua morte. Pela via de seu racionalismo, o pensamento de Bachelard tem uma importante influência na epistemologia francesa contemporânea. Pela via do devaneio poético, o bachelardismo ressurge no Séc. XXI por meio de interpretações artísticas, geográficas e arquitetônicas. O resultado dessa exposição visa então posicionar o leitor no que o autor chama de uma dupla cultura, na oposição e complementariedade das duas faces do espírito, anima e animus, dando ao ser humano a profundidade do ser e os meios de uma liberdade, verdade e felicidade. Ao mesmo tempo, se desdobra desse bachelardismo três novas perspectivas: educação, crítica literária e ecologia, como novas faces da atualidade do pensamento desse grande pensador trans-disciplinar que perpassa do corpo ao intelecto, dos afetos às configurações sociais. A crítica literária nos leva à nova hermenêutica e fenomenologia que circulam em torno dessa pedagogia estética da inteligência; por fim, uma ecologia das paisagens, na qual a imaginação é um geopathos, ou seja, um amor pela terra e por se entender como parte integrante e atuante dos ecossistemas num biomimetismo simbiótico entre o ser humano e a natureza.

Palavras-chave: Gaston Bachelard. Legado contemporâneo. Teoria-prática. Dupla cultura.

\section{INTRODUCTION}

L'apport de Bachelard relève des deux grands domaines de l'expérience humaine, longuement décrite, valorisée et renouvelée : la culture scientifique, la science, et la culture poético-artistique, la rêverie.

Sa défense et sa promotion d'une science pure, libérée de l'imagination, des idéologies, de ses déterminations économiques, entrainée par une rationalité dialectique dynamique et abstraite laisse en héritage un rationalisme fort, qui tranche avec les relativismes constructivistes et sociologisant, issus du Cercle de Vienne ou de l'épistémologie anglo-saxonne. Cet idéal, même malmené par les réceptions historiques, 


\section{BACHELARD, ETAT DES LIEUX 55 ANS APRES SA MORT}

\section{Jean-Jacques Wunenburger}

demeure un horizon du rationalisme français et même européen, avec ses accents positivistes voire scientistes parfois.

Quant à la place et à la richesse de l'imagination poétique, Bachelard lui a consacré de plus en plus de travaux originaux, difficiles à classer, mais qui lui ont valu l'intérêt et la reconnaissance de tous les créateurs, du danseur à l'architecte. Il a ainsi mis en place une pédagogie, épistémologie, esthétique et même éthique de l'imagination créatrice, qui inspire un grand nombre de discours et pratiques de l'innovation et du changement.

L'épistémologie bachelardienne a donné naissance d'abord à un style spécifiquement français de travaux sur l'histoire des sciences, qui sont marqués par les noms de Georges Canguilhem, Louis Althusser, Michel Foucault ou François Dagognet. Bachelard sert souvent de référentiel majeur pour définir un certain type d'épistémologie française, héritière sans doute d'un rationalisme spéculatif du concept, parfois teinté d'un idéalisme plutôt germanique (Hegel), même s'il a fait une large place à la matériologie et à la technologie expérimentale. Sans doute cette épistémologie estelle concurrencée aujourd'hui par les écoles issues de Cercle de Vienne, de la philosophie analytique anglo-saxonne mais aussi par différentes approches plus sociologiques et relativistes qui mettent l'accent sur la construction économique et politique des sciences. Il reste que Bachelard demeure une référence majeure pour orienter et légitimer une pédagogie de l'esprit scientifique, qui se trouve toujours confrontée aux relations complexes entre l'esprit concret et l'esprit abstrait, entre le formalisme et le pragmatisme.

Quant au Bachelard poéticien, il a servi de miroir agrandissant aux artistes de nombreux arts (des arts plastiques à l'architecture et à la danse) qui ont reconnu dans ses analyses des vérités intimes sur leur propre imagination créatrice et qui a laissé son empreinte sur des critiques littéraires et d'arts plastiques, français et étrangers. Par ailleurs il sert de référence autant à des approches quasi formalistes et structuralistes, mises en œuvre au début, qu'à une tradition de psycho-critique, pour laquelle les œuvres langagières - poétiques plus que narratives - doivent leur profondeur et leur nouveauté à des structures symboliques immanentes, ouvrant, dans la création comme la réception, 


\section{BACHELARD, ETAT DES LIEUX 55 ANS APRES SA MORT}

Jean-Jacques Wunenburger

à une expérience existentielle, à une intériorisation qui ravive les images les plus anciennes et archétypiques (de Jean-Pierre Richard à Michel Collot).

Enfin, il ne faudrait pas négliger que le bachelardisme resurgit au début du XXIème siècle, dans bien des approches phénoménologiques, non quantitatives, de l'espace, chez des géographes ou des architectes, sensibles à son approche vécue et symbolique des formes et matières; de même il rejoint souvent, aux côtés de Heidegger et Léopold, les tenants d'une critique écologique du monde de la domination et de l'exploitation technique et scientifique au profit d'un respect de la nature, inséparable de son imaginaire. La poétique matérielle est un inducteur d'intensification ontologique pour le sujet et participe à «l'esprit des lieux », à cette subjectivation de l'espace qui en fait en même temps une dilatation, une matérialisation du Moi. Bref la matériologie onirique bachelardienne devient un partenaire d'un réenchantement du monde.

\section{L'APPORT SCIENTIFIQUE}

Bachelard a développé ses études de la rationalité scientifique dans les années 1920 dans le sillage des grandes révolutions scientifiques (depuis 1905), les géométries non euclidiennes, la relativité (Einstein), la mécanique quantique (école de Copenhague), qui le conduisent à s'engager dans une réinterprétation, à contre-courant de la pensée dominante, de la rationalité scientifique. Bachelard appartient à ce moment de la modernité du XXème siècle où, en France en particulier, les philosophes (H. Bergson, E. Brunschvicg, E. Meyerson etc.) réinvestissent le savoir scientifique qui est en train de bouleverser - mais tous, même en sciences, ne l'ont pas compris tout de suite -, la représentation du monde mais aussi les manières de le comprendre, qui ne se satisfont plus ni du kantisme, ni du positivisme (Cournot, Renouvier, Meyerson, Duhem).

On peut trouver chez Bachelard, dans ses nombreux livres qui s'étalent entre 1927 et 1953, - qui ne se répètent pas, car Bachelard avance toujours dans la pensée et dans sa langue vers des formulations rectifiées - tous les grands thèmes de l'histoire des sciences et de l'épistémologie critique qui vont marquer les débats de la rationalité des sciences du siècle: démarcation entre savoir non-scientifique et savoir scientifique, 


\section{BACHELARD, ETAT DES LIEUX 55 ANS APRES SA MORT}

Jean-Jacques Wunenburger

dialectique théorie-expérience, phénoménotechnique, construction sociale de la science, mais déclinée à sa façon particulière, maintenue dans les limites d'une confiance en la raison qui n'est pas infidèle aux Lumières. De ce point de vue, Bachelard peut être tenu pour un précurseur aussi bien de la catégorie de techno-science que de la sociologie historique des sciences, même s'il n'a pas vu la place de l'économie et du politique dans l'appareil industriel de la science et s'il a refusé de céder aux tentations du relativisme, propres aux constructivistes ultérieurs (S. Woolgard, B. Latour, Feyerabend). T. Castelao tient à montrer la voie équilibrée que représente pour G. Bachelard un rationalisme dialectique, à égale distance des courants irrationalistes et relativistes d'une part, et des tenants des formalismes, logiques analytiques et autres cognitivismes, d'autre part. Elle focalise l'originalité de G. Bachelard sur le concept de "phénoménotechnique" (1931), qui illustre et récapitule, à travers ses variations, sa conception de la science. Très tôt familier de la physique mathématique, à la suite de E. Brunschvicg, qui lui fait défendre la valeur méthodologique des mathématiques, Bachelard n'en valorise pas moins - dans une circularité nommée dialectique - le moment réaliste et matériologique, où la raison provoque la réalité par l'expérimentation, elle-même fruit d'une théorie. Mais cette épistémologie de la mobilité entre abstrait et concret, entre théorie et pratique, n'en n'a pas moins le souci d'inscrire la science dans une histoire qui la contraint à des ruptures permanentes, et dans une société, qui a la charge d'éduquer l'esprit à la science et de lui fournir une éthique de la discussion et de l'intersubjectivité. La fameuse vérité objective de la science, qui reste la valeur incontestable, résulte donc d'une concomitance complexe de conditions et de facteurs pluriels, une dynamique interne de la raison s'auto-rectifiant, un inter-rationalisme de surveillance mutuelle, une construction de concepts - parfois antithétiques - de plus en plus abstraits, mais par le moyen d'artefacts expérimentaux, une ouverture aux révolutions le plus profondes des théories en renonçant à toute absolutisation des représentations au profit d'une raison approchée. 
BACHELARD, ETAT DES LIEUX 55 ANS APRES SA MORT

Jean-Jacques Wunenburger

\section{L'APPORT POETIQUE}

Dès son premier ouvrage consacré à l'imaginaire, combattu dans la rationalité mais source de la poétique, La Psychanalyse du feu (1938), Bachelard convoque conjointement la psychanalyse freudienne (qui lui apparaîtra plus tard comme réductrice) et la psychologie des profondeurs de C.G. Jung. A partir d'une herméneutique symbolique des matières, il décrit les processus imaginatifs qui traversent le sujet en connectant son histoire personnelle (particulièrement son enfance rêvée) à des réseaux de signification d'images transindividuelles, dont témoignent les mythes. Par là, Bachelard développe progressivement une psychologie de la création, qui retrouve l'inspiration de la « fantastique transcendantale » chère au poète allemand Novalis.

Certes Bachelard répète souvent qu'il faut « expliquer les rêves par les rêves », ce qui ne l'empêche pas, dès La Psychanalyse $d u f e u$, d'annoncer un projet de rationalisation de l'imaginaire, de véritable science de la création poétique, dont la syntaxe et la sémantique seraient formulables par des lois. Si Bachelard a, par la suite, renoncé à systématiser une logique des images pour lui substituer une approche plus phénoménologique, influencée par Ed. Husserl, c'est encore pour saisir des conditions formelles et transcendantales de la rêverie.

La créativité de l'imagination, au sens d'une faculté de déformer les images, apparaît donc comme rationalisable, du fait de propriétés clairement identifiables :

- d'abord, les images, loin d'être toutes des résidus perceptifs passifs ou nocturnes, se présentent souvent comme des représentations dotées d'une puissance de signification et d'une énergie de transformation. Proche des analyses de C.G. Jung, Bachelard situe les racines de l'imagination dans des matrices inconscientes (les archétypes), qui se dissocient elles-mêmes en deux polarités, masculine (Animus) et féminine (Anima), qui modifient le traitement des images soit dans un sens volontariste de lutte, soit dans un sens plus pacifique de réconciliation. Loin d'être refoulées, comme pour Freud, ces images sont ensuite transformées par une conscience onirique en images nouvelles au contact des éléments matériels du monde extérieur; 


\section{BACHELARD, ETAT DES LIEUX 55 ANS APRES SA MORT}

Jean-Jacques Wunenburger

- l'imagination ne saurait être assimilée à une activité seconde, dérivée de la perception, dont elle combinerait seulement les matériaux, ni à un simple jeu fantaisiste avec des images. Au contraire, l'imagination véritable travaille sur des images a priori, indépendantes et même antérieures aux représentations de la perception;

- les images se chargent de significations symboliques nouvelles, non subjectives, au contact des substances matérielles du cosmos qui leur servent de contenu privilégié. Elles s'enrichissent et se nourrissent en effet de la symbolique des quatre éléments (feu, eau, air et terre), qui fournissent des «hormones de l'imagination », qui nous font «grandir psychiquement». Ainsi l'imagination, si elle est bien profondément liée à l'inconscient personnel du rêveur, se présente avant tout, du point de vue de son contenu, comme une imagination matérielle, dont les rêveries le relient intimement au cosmos; - cette force créatrice d'images est elle-même sous-tendue, comme chez Schopenhauer, par une volonté de vivre, un vouloir-vivre primitif, principe de la vie spirituelle. Car l'imagination est d'abord au service d'une énergie vitale qui lui confère une causalité créatrice et qui la met au service de ses rythmes propres de poussée et de détente. C'est pourquoi elle oscille toujours entre mouvement et repos, entre extraversion et introversion, qui constituent les deux polarités fondamentales du psychisme, sources de deux régimes bien distincts d'activités imaginantes; l'imaginaire du monde, naturel comme construit (celui de la maison natale si chère à Bachelard) dérive de trois caractères: formel, matériel et dynamique. Si Bachelard minimise souvent l'importance de l'imaginaire des formes des objets, trop rationalisables, il s'attache longuement à l'imagination matérielle (5 volumes sur les matières), et de plus en plus, à l'imagination dynamique, qui épouse le plus intimement l'activité du psychisme. Il reste que la puissance de rêverie des formes pures, même géométriques, a été explorée et exaltée dans La Poétique de l'espace, où Bachelard dévoile des ressorts puissants de la poétique de la nature mais aussi des objets fabriqués et même de l'architecture; - enfin les images trouvent leur dynamique créatrice dans l'expérience du corps propre, par exemple la parole ou le travail musculaire à travers ses mouvements, ses rythmes, la résistance des matières travaillées par le geste, et finalement la conscience temporelle discontinue, qui est faite d'instants successifs et novateurs, entraînés par un rythme. 


\section{BACHELARD, ETAT DES LIEUX 55 ANS APRES SA MORT}

Jean-Jacques Wunenburger

\section{LA DOUBLE CULTURE}

Mais c'est finalement l'opposition et l'unité complémentaire des deux faces de l'esprit et des deux cultures qui définit la vocation de l'homme complet et qui détermine sa relation heureuse et même sa sagesse de bien vivre. Car Bachelard expose bien deux voies opposées de développement de l'esprit humain, l'une qui passe par la formation à la connaissance scientifique, l'autre par la créativité onirique et poétique. Certes Bachelard applique aux démarches de la rationalité et de l'imaginaire des catégories descriptives et normatives semblables: entre autres, un énergétisme originaire, hostile à tout blocage sur des représentations, une dialectique des concepts comme des images, qui implique une auto-générativité de l'image et du concept par un jeu d'oppositions (la contradiction pour la raison, les polarités contraires pour l'imagination) etc. Mais Bachelard voit bien dans ces deux modes de pensée et de vie des dimensions antagonistes et complémentaires comme le jour et la nuit, chacune devant occuper l'esprit pour moitié. L'homme ne se réalise qu'en alternant les deux cultures, sans renoncer à l'une d'elles. En s'adonnant à l'une et à l'autre l'homme s'ouvre à une profondeur d'être, et se donne les conditions et les moyens d'accéder à la vérité et à la liberté, sources de tout bonheur.

Cette réalisation des deux dispositions de l'esprit participe en même temps d'une éthique, un art du bien vivre et finalement une sagesse. Car en cultivant la rigueur austère de la rationalité scientifique et la créativité exubérante de la rêverie poétique, chacun se sent appelé à se surmonter, à tourner le dos aux forces morbides, régressives et obscurantistes et à intensifier l'existence, ce qui peut se nommer une éthique. Bachelard fait donc de la science et de la poésie deux voies fondamentales qui expriment la richesse et la vitalité de l'esprit humain. Par la voie du concept comme par celle de l'image, l'esprit recommence à chaque fois une aventure de dépassement de soi et de création d'œuvres inédites, qui témoignent de sa véritable destination. Bachelard nous invite bien à poser que chaque jour de notre vie, qui a 24 heures, nous pouvons nous réaliser de manière duelle au moins. Il est des situations, des heures, des saisons où l'homme se reconnait dans des obligations impératives, dans d'autres où il aspire à se réaliser dans ses désirs et rêveries. 


\section{BACHELARD, ETAT DES LIEUX 55 ANS APRES SA MORT}

Jean-Jacques Wunenburger

\section{LES PROLONGEMENTS ET HERITAGES}

Dans le sillage de cette œuvre double, les idées de Bachelard ont irrigué bien des domaines d'application. On en retiendra trois: la formation et l'éducation, la critique littéraire et l'écologie: - Education: L'homme bachelardien se révèle doté d'une nature complexe, qui mobilise toutes les dimensions du sujet, du corps à l'intellect en passant par l'affectivité, la volonté et l'imagination. Il s'enrichit selon plusieurs vocations, modes de réalisation, d'abord la pensée commune, adaptée, socialisée, puis le travail ascétique de la connaissance scientifique, enfin l'épanouissement hédoniste de la rêverie du monde, chacune de ces dimensions éveillant en lui des valeurs propres qui constituent autant de visages d'une éthique plurielle. Dans toutes ces configurations, l'homme est invité à se laisser entraîner à un devenir, le long d'une verticalité qui lui fait conquérir sans cesse des nouveautés procurant vérités, vertu et bonheur. Mais cette progression continue tous azimuts reste scandée par des rythmes d'alternances, de féminité et de masculinité («animus » et «anima»), de repos et de travail, d'intériorisation et d'extérioration, dessinant ainsi une harmonie vibrante de pôles alternatifs. De la sorte, il semble que Bachelard plaide à travers le miroir de son œuvre épistémologique et esthétique pour une sagesse humaine, délaissée depuis longtemps, en tout cas depuis l'antiquité et la Renaissance. Tout en ne sacrifiant pas les meilleurs acquis du rationalisme des Lumières ni l'héritage du romantisme allemand, il a mis en œuvre une sorte de synthèse originale des Lumières et du romantisme, cherchant à réconcilier en l'homme les contraires du jour et de la nuit, tout en engageant toujours l'homme intégral dans ses métamorphoses de soi. Aux absolutisations intempérantes du rationalisme impérialiste, il a opposé un homme bi-frons, tel Janus aux deux visages, tout en faisant de cette dualité une totalité rythmée, harmonieuse et bienfaisante. Il ne semble pas qu'il existe dans la philosophie moderne d'aussi ambitieuses et justes conceptions de l'homme, aussi soucieuses d'équilibres entre les opposés, que celle de G. Bachelard; - critique littéraire: à travers une œuvre consacrée pour moitié à une philosophie de l'imagination créatrice et de l'imaginaire poétique, Bachelard n'a cessé de manière éclectique et empirique de convoquer, rendre opératoire et transformer toutes sortes d'outils, de méthodes et d'écoles en développement en son temps. Tour à 


\section{BACHELARD, ETAT DES LIEUX 55 ANS APRES SA MORT}

Jean-Jacques Wunenburger

tour psychologie, psychanalyse, symbolologie, structuralisme, phénoménologie servent à rendre compte de la consistance profonde des imaginaires et de leur nouveauté et créativité. Il reste qu'un pan de l'œuvre est dominé par un souci de redonner toute sa place au vécu, à l'expérience des mots, des images et des symboles dans leur rencontre mentale, perceptive et verbale. Mais ce moment de dévoilement d'images, toujours dotées d'une consistance ontologique, n'est là que pour permettre d'en déployer, d'en exhiber les potentialités symboliques. Toute image dans son expérience poétique même est une ouverture à des sens multiples mais profondément liés à des grammaires symboliques. Et ces réseaux de connotations symboliques renvoient à l'être imaginant, à sa condition, à ses conduites, à ses aspirations et idéaux psychologiques, éthiques et même métaphysiques ${ }^{2}$. Par-là l'œuvre de Bachelard se tient en un carrefour où se rencontrent les grands acquis de la phénoménologie et de l'herméneutique en une alliance originale et toujours en mouvement. Bachelard ne cherche pas à formuler un système de critique littéraire mais au fur et à mesure de son expérience de l'imaginaire, il rencontre des questions et des problèmes, qui sont autant de points de passage obligés, sur lesquels il porte un jugement d'expérience, qui peut valoir orientation voire prescription. Bachelard rencontre ainsi la plupart des questions de la créativité et de l'herméneutique, prend position, empiriquement, subjectivement, mettant ainsi en place une conception bachelardienne de l'imagination et de l'imaginaire. Si on ne peut la considérer comme une méthode achevée et argumentée, ce qu'elle se refuse même, sa poétique illustre une certaine approche des textes, qui peut aussi bien être adoptée et prolongée, que mise en débat voire critiquée. De ce point de vue, Bachelard par ses goûts et propositions théoriques constitue une entrée critique et pédagogique sur les questions de toute critique littéraire. Mais cette théorie bachelardienne de la littérature reste sans doute encore à écrire; - ecologie: par son approche singulière de la poétique des éléments cosmologiques, Bachelard s'engage dans une vision du monde holistique et dynamique, qui resurgit aujourd'hui dans le paradigme écologique. En lieu et place d'un dualisme entre homme et nature, entre sujet et monde, entre esprit et matière, Bachelard décrit et valide dans sa psychologie de l'imagination une sorte de dialectique

\footnotetext{
${ }^{2}$ Voir J.J. Wunenburger (s. dir.), Gaston Bachelard, science et poétique. Pour une nouvelle éthique ?, Hermann, 2013
} 


\section{BACHELARD, ETAT DES LIEUX 55 ANS APRES SA MORT}

Jean-Jacques Wunenburger

entre l'extérieur et l'intérieur, entre le mécanique et le vivant qui sous-tend notre rapport vécu au monde. Bachelard et l'épistémologie de l'écologie contemporaine renoncent en effet à prendre appui sur la seule Nature homogène, unidimensionnelle, construite par la science. Qu'est-ce, en effet, que la Nature si ce n'est l'ensemble des éléments primordiaux (Archai), des lieux (topoi) et des milieux, qui n'existent et ne se disent qu'au pluriel. Bachelard retient l'idée que chaque individu, groupe ou société ne vit et ne se développe que dans un milieu idiosyncrasique, un paysage, qui est aussi son pays, sa demeure (oikos). La condition d'un rapport poético-écologique ne vient pas d'un même traitement de la Nature mais de ce que les habitants vivent dans une certaine « ambiance » (Stimmung), où l'homme et le milieu procèdent sans cesse à des échanges. Pour que les hommes puissent donc se sentir habitants d'un monde, il leur faut donc renoncer à une science abstraite universelle comme à une sacralisation de leur milieu, qui les isolerait de l'histoire et de la vie; l'essentiel, au contraire, réside dans un art de percevoir et d'imaginer, d'une esthétique, qui permettent de reconnaître un «monde », un milieu spécifique (analogue au «monde perceptif» de Uexküll, qui est condition pour que l'homme ait prise sur la terre et pour qu'il y ait emprise de la terre sur l'homme). Mais il faut aussi, pour que l'homme puisse prendre appui sur son milieu et puisse le prendre en main, qu'il soit capable de le com-prendre (au sens de prendre en fermant la main), qu'il y ait emprise du monde sur l'homme, ou que l'homme éprouve une sym-pathie, une intro-pathie à l'égard du monde. A cet effet, il faut suspendre les rapports d'objectivation avec l'environnement, traité comme un ensemble de choses inertes, et se détourner des seules régressions intimistes, qui ne voient dans le milieu que des espaces étrangers voire aliénants. Vivre par la rêverie «avec»-plus que « dans » - son paysage, ce n'est ni lui imposer des modèles abstraits arbitraires, ni le rejeter dans une valence négative, c'est croître ensemble avec lui. Telle est la fonction d'une participation onirique à l'éco-système, à travers laquelle seulement on peut parfaire sa propre individuation. Et pour Bachelard nulle plus que l'imagination n'est à même de nous faire participer ainsi au monde dans lequel nous rêvons. L'écologie imaginative implique donc une géo-pathie, une capacité à ressentir un paysage, à « consentir » à un lieu, à vibrer avec lui, à descendre dans ses profondeurs par les images, mais pour mieux avoir prise sur lui, et non seulement pour le connaître froidement. Pour 


\section{BACHELARD, ETAT DES LIEUX 55 ANS APRES SA MORT}

\section{Jean-Jacques Wunenburger}

cela, l'habitant et son pays doivent «entrer en phase », être sur la même longueur d'onde, avoir le même pli. C'est pourquoi les activités humaines créatrices reposent fréquemment sur une intériorisation des rythmes du paysage (au sens spatial et temporel de la découverte des intervalles entre des formes) et sur une extériorisation des rythmes corporels dans le monde extérieur. Comme Bachelard l'avait établi dans ses travaux sur le temps instantané et rythmique, la relation entre l'homme et la nature doit être, en ce sens, rythmophasique, ce qui exige un imaginaire de type «copulatif», et non assimilatif ou digestif (qui introjecte l'extérieur dans l'intérieur), ni adaptatif ou postural (où l'intérieur domine l'extérieur pour se le soumettre). Dès lors, la vie interactive est constamment «re-jeu», c'est-à-dire mimétisme symbiotique entre l'extérieur et l'intérieur, mais sans suppression de l'identité spécifique et de la singularité réciproque de la nature et de l'homme. 